\title{
Nuevas vías para la comunicación empresarial: publicidad en el móvil
}

\section{Por Claudio Feijóo-González, José-Luis Gómez-Barroso e Inmaculada J. Martínez-Martínez}

\begin{abstract}
Resumen: La comunicación empresarial en general y la publicidad en particular están necesitadas de estrategias más ajustadas y eficaces. Se presenta la publicidad en dispositivos móviles y se analizan sus rasgos diferenciales, basados en la información personal y de contexto que podría utilizarse. Se analiza su posible evolución a partir del estudio de los factores económicos, tecnológicos y sociales que la van a condicionar a corto y medio plazo.
\end{abstract}

Palabras clave: Publicidad, Comunicaciones móviles, Audiovisual, Contenidos, Acceso a internet, Terminales móviles.

Title: New opportunities for business communication: mobile advertising

Abstract: Business communication in general, and advertising in particular, is in need of more targeted and effective approaches. This article introduces mobile advertising and its differential features, including the role of contextual advertising and customized relationship with mobile devices. It analyses future potential, identifying essential economic, technological and social forces that will shape mobile advertising in the short and middle term.

Keywords: Advertising, Mobile communications, Audiovisual, Mobile content, Internet access, Mobile terminals.

Feijóo-González, Claudio; Gómez-Barroso, José-Luis; Martínez-Martínez, Inmaculada J. “Nuevas vías para la comunicación empresarial: publicidad en el móvil”. El profesional de la información, 2010, marzo-abril, v. 19, n. 2, pp. 140-148.

DOI: 103145/epi.2010.mar.04
Claudio Feijóo-González es doctor ingeniero de telecomunicación por la Universidad Politécnica de Madrid donde es profesor e investiga el impacto socioeconómico de la evolución de tecnologías emergentes ligadas con el progreso de la sociedad de la información. En este ámbito trabajó en el Institute for Prospective Technological Studies (IPTS) de la Comisión Europea. Dirigió la Cátedra COIT-UPM en Regulación y Políticas Públicas relativas a la sociedad de la información, ha sido asesor de la Secretaría de Estado de Telecomunicaciones y para la Sociedad de la Información, y ha participado y dirigido múltiples proyectos de investigación. Es autor de numerosas publicaciones internacionales y conferenciante y profesor en varios cursos de postgrado.

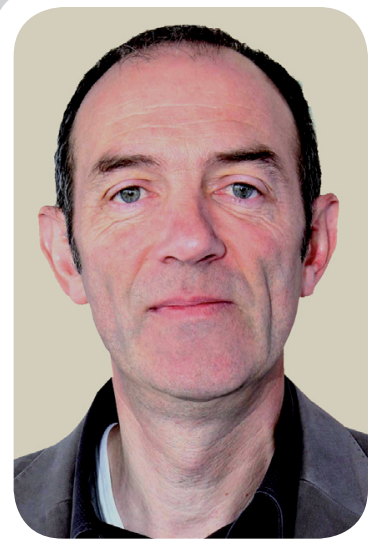

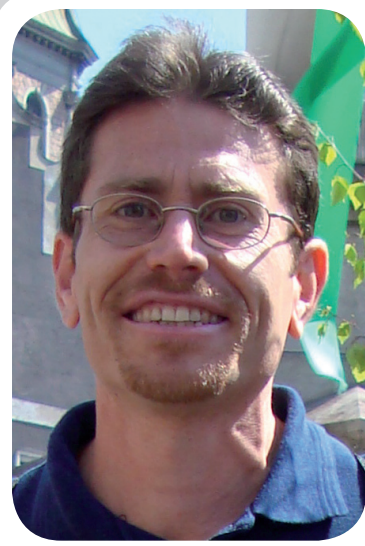

José-Luis Gómez-Barroso es profesor del Departamento de Economía Aplicada e Historia Económica de la Universidad Nacional de Educación a Distancia (UNED). Es doctor y licenciado en ciencias económicas y empresariales por la UNED, ingeniero de telecomunicación por la Universidad Politécnica de Madrid y licenciado en derecho por la Universidad Complutense. Ha participado en convenios y proyectos financiados por instituciones nacionales y por la Comisión Europea. Ha sido profesor invitado en la Universidad de Columbia, la Universidad de Florida, la École Nationale Supérieure des Télécommunications, la Università La Sapienza, el Institut National des Télécommunications, y la Fondazione Ugo Bordoni.

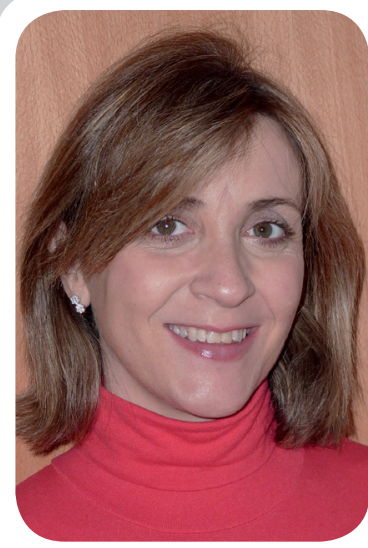

Inmaculada J. MartínezMartínez es doctora en ciencias de la información por la Univ. Complutense de Madrid y master en dirección y gestión de empresas por la Know How Business School. Ha realizado consultoría y formación incompany sobre estrategias de imagen y comunicación en España, Brasil y Portugal. Miembro fundador del Grupo de Investigación en Comunicación Social, Cultura y Tecnología (E-COM), en el que participa en los proyectos Movilsoc (Impacto social de la telefonía móvil en España) y Mobile Media (Evolución del medio móvil en España). Dirige el proyecto I+D sobre mujer y tecnologías móviles Movilizad@s. Es profesora de publicidad y relaciones públicas en la Facultad de Comunicación y Documentación de la Univ. de Murcia.

\section{Introducción}

\section{LA COMUNICACIÓN EMPRESARIAL} ABARCA las diversas vías mediante las que una organización se comunica con todos aquellos agentes (inversores, clientes, suministradores, empleados...) con quienes la compañía se relaciona o podría hacerlo.

Parte de esta comunicación es controlada por la propia empresa y parte no lo es, pues su actividad genera espontáneamente diferentes percepciones entre 
esos agentes (Melewar; Karaosmanoglu, 2006). La comunicación empresarial, controlada o dirigida, adquiere rasgos diferentes en función de cuál sea el objetivo al que se dirige (Argenti, 1996): comunicación con (o para) empleados, con el sector público, dirigida al sistema financiero o a los medios de comunicación y por supuesto, como parte fundamental de esta comunicación, publicidad destinada a los posibles clientes ${ }^{1}$.

Muchos modelos que han utilizado las empresas en las últimas décadas para llevar a cabo estas modalidades de comunicación están sometidos a grandes transformaciones y algunos se hallan en crisis. En particular la publicidad difundida en medios de comunicación ha reducido su impacto significativamente. Dos razones básicas explican este hecho. La primera es la creciente fragmentación de la audiencia, debida a su vez a la multiplicación de ofertas para el acceso a la información y para el disfrute del ocio, factores que impulsan un cambio en los hábitos sociales. La segunda es la sobreexposición a la publicidad ${ }^{2}$, de modo que el público sólo retiene unos pocos mensajes que realmente captan su interés.

\section{"Se requieren nuevos métodos con que identificar y llegar al público"}

Ante esta situación hay dos retos que hay que afrontar para lograr una comunicación más efectiva. En primer lugar, se requieren nuevos métodos con los que identificar y llegar al público, y en segundo lugar, el mensaje debe ajustarse mucho más al perfil del receptor al que va dirigido.

Parece obvio que una variable clave para resolver ambas ecuaciones son las tecnologías de la información y la comunicación (TIC) tanto por las posibilidades técnicas que ofrecen como por las transformaciones sociales que están originando. Internet ha abierto nuevas vías de comunicación que ya son usadas aunque en muchos casos aún de manera rudimentaria. La evolución permanente de las TIC permitirá profundizar y perfeccionar las estrategias hasta ahora utilizadas y crear otras innovadoras.

Parte de esta evolución está ligada con la forma en que se accede a contenidos y aplicaciones. El teléfono móvil o, con más rigor, los terminales válidos para la recepción de comunicaciones móviles ${ }^{3}$, está ocupando progresivamente un papel central como dispositivo con el que "conectarse con el resto del mundo", acaparando otras funciones más allá de la mera transmisión de $v z^{4}$. Esta circunstancia tendrá repercusiones en diver- sos ámbitos e indudablemente debe ser tenida también en cuenta por los responsables de la comunicación y publicidad empresarial.

\section{"El mensaje publicitario debe ajustarse mucho más al perfil del receptor al que va dirigido"}

En efecto, el móvil posee unas características que lo hacen particularmente interesante. Se encuentra habitualmente conectado de forma permanente y el usuario lo lleva consigo a todas partes. Además es un aparato mucho más personal que un ordenador y en cada llamada o conexión es posible identificar no sólo quién la realiza sino dónde se encuentra. Son evidentes las opciones que ello ofrece a los responsables de comunicación que puedan manejar sólo parte de esa información. Siguiendo la regla de oro de destinar recursos donde el impacto potencial sea mayor, el futuro de la publicidad en el móvil se promete brillante.

Este artículo realiza un repaso del estado y condiciones para el avance de "la publicidad móvil"s. En el siguiente apartado se caracteriza el mercado, analizando la cadena de valor y las modalidades y estrategias de comunicación; a continuación se presentan los factores que hacen del móvil un vehículo atractivo para la publicidad de las empresas y también se estudian las barreras que podrían frenar su evolución; y por último, cierran el trabajo unas conclusiones.

Cabe puntualizar que nos referiremos a la publicidad incluida en servicios recibidos en el móvil que no son de telefonía, aunque también en este ámbito han existido algunas experiencias ${ }^{6}$.

\section{Caracterización del mercado de la publicidad móvil}

\section{Cadena de valor}

Los mercados de servicios y aplicaciones relacionados con las comunicaciones móviles han evolucionado desde situaciones de dominio de un único tipo de agente (el operador de comunicaciones) a otras que pueden caracterizarse como un ecosistema complejo en que un número elevado de intervinientes colabora y compite a un mismo tiempo.

En este ecosistema la actividad empresarial, como en cualquier otro mercado TIC (Fransman, 2007), puede agruparse en tres grandes categorías:

- elaboración/producción de contenidos y aplicaciones 
- distribución

- uso/consumo/interacción

La figura 1 muestra los agentes que intervienen en el complejo proceso mediante el que las compañías llevan mensajes a sus clientes potenciales. Las funciones de estos agentes son claras con excepción probablemente de dos de ellas. Se ha denominado "facilitadores" a aquellas empresas cuya función es poner en contacto a anunciantes con los proveedores de contenidos y aplicaciones (dueños de sitios web, fabricantes de videojuegos...). Por su parte, el papel de los "adaptadores" es permitir que las comunicaciones/publicidad procedente de diversas fuentes e insertada en diferentes medios esté a disposición de los usuarios, con independencia del operador de comunicaciones móviles al que estén suscritos y del dispositivo móvil que posean. En ambos casos, estas empresas suelen suministrar además diversas aplicaciones para el seguimiento de la publicidad efectuada y para evaluar su potencial impacto.

Cada operación no ha de ser llevada a cabo necesariamente por una empresa independiente. De hecho, muchas compañías pugnan por integrar la mayor cantidad de actividades posibles de las de esta cadena de valor. Cuando un determinado agente tiene éxito con estas estrategias e integra varias de las actividades clave tratando de controlar el resto, forma lo que se denomina "plataforma". Son las plataformas de los operadores de comunicaciones móviles las que durante años han impuesto las condiciones con las que ha evolucionado el mercado de la publicidad móvil. En los últimos años, su papel se ha debilitado y han aparecido otras nuevas, particularmente las de determinados fabricantes de teléfonos (terminales) móviles?

No sólo la integración vertical de eslabones de la cadena de valor en una plataforma sino también la integración horizontal de las diferentes compañías presentes en uno de esos eslabones, puede permitir a una empresa determinada el establecimiento de una especie de puesto de control (más bien de peaje) para el resto de agentes ${ }^{8}$.

Las buenas perspectivas anunciadas para el mercado de la publicidad móvil están en el origen de la consolidación e integración de su cadena de valor, proceso en que están participando algunas de las mayores empresas del sector TIC. Así, desde 2006 y en una operación que parece haberse acelerado recientemente, algunas pequeñas empresas que habían destacado en algún aspecto de la publicidad móvil han sido adquiridas por grandes como Google (adquisición de AdMob), Microsoft (adquisición de ScreenTonic), Apple (Quattro Wireless), AOL (Third Screen Media), Yahoo (Actionality), Nokia (Enpocket), o Vodafone y Telefónica Móviles (quienes curiosamente invirtieron simultáneamente en la compañía Amobee).

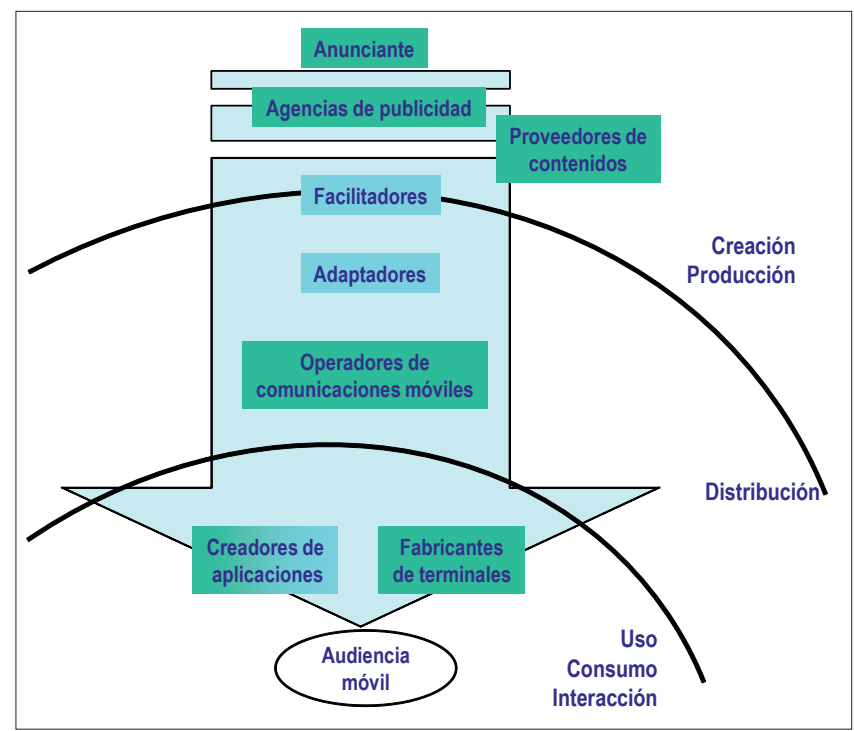

Figura 1. Cadena de valor de la publicidad móvil (adaptada de Feijóo-González et al.,, 2010)

\section{Modalidades de comunicación}

La promoción por teléfono móvil puede transmitirse de dos formas:

- La primera, que coincide con la publicidad tradicional, puede denominarse comunicación indiferenciada, pues no hay otra adaptación o discriminación del mensaje más allá de la que tiene su origen en la segmentación del público al que se dirige.

- La segunda toma en cuenta las opciones de personalización que las comunicaciones móviles ofrecen. Por un lado puede aprovecharse la información de contexto ya que en una comunicación móvil siempre es posible conocer con cierta exactitud dónde se encuentra el usuario9 ${ }^{9}$. Por otro lado puede manejarse información personal pues el móvil es un dispositivo con el que los usuarios mantienen una relación muy personal y podrían con facilidad extraerse datos con que modelar su perfil socioeconómico y de comportamiento (si el propio usuario no impone restricciones) ${ }^{10}$.

\section{"La publicidad no discriminada es más propia de portales genéricos o de medios de comunicación"}

Las dos variantes descritas son tecnoeconómicas pues en la elección de una u otra intervienen decisiones tácticas pero también requieren diferentes avances tecnológicos, claramente más complejos (y no siempre disponibles) en el segundo de los casos.

La publicidad no discriminada es más propia de portales genéricos o de medios de comunicación. En 
su versión móvil se ha basado fundamentalmente en el envío de mensajes cortos, incluyendo la posibilidad de cierta interactividad $^{11}$. Es el medio más extendido de publicidad móvil, dada la adopción de la mensajería corta por prácticamente todos los usuarios de comunicaciones móviles.

La publicidad orientada tiene diferentes modalidades en función de la cantidad de información que se maneje. Algunas de las tácticas del internet fijo son por supuesto válidas en el sector del móvil. De hecho, dejando a un lado ciertas especificidades del medio (como el envío de mensajes cortos antes mencionado), hasta ahora las estrategias para la publicidad en el móvil han venido siendo tributarias de la publicidad en el internet fijo (véase a este respecto un análisis general en DelPino-Romero, 2010).

En el escalón inferior pueden citarse por ejemplo los "enlaces patrocinados" en los resultados de una búsqueda, cuyo mercado crecerá a medida que los buscadores móviles evolucionen ${ }^{12}$. Si se sabe algo más del usuario (porque se conocen datos sobre la navegación que ha realizado o porque haya completado un cuestionario), la publicidad puede ser más afinada.

Y en un tercer escalón, el realmente asociado con la movilidad que une datos personales y datos de contexto, se pondría a disposición del usuario publicidad de por ejemplo los restaurantes que se hallan cercanos al lugar donde se encuentra y que cumplen con sus restricciones presupuestarias o con sus gustos. No es difícil imaginar que en este último escenario sea el propio usuario el que demande dicha información. Publicidad solicitada, cuyo impacto es indudable, es el sueño de todo publicista.

\section{"La publicidad orientada tiene diferentes modalidades en función de la cantidad de información que se maneje"}

Ambas modalidades, indiferenciada u orientada, son compatibles con las diferentes formas con que se hace llegar la promoción a los usuarios. Como norma general, recibir publicidad es la contrapartida por el acceso libre a algún tipo de información, contenido ${ }^{13}$, servicio o aplicación ${ }^{14}$.

La promoción puede aparecer como un recuadro (banner) colocado en algún lugar de la propia información, como una pantalla entera (visible frecuentemente antes de acceder a lo que se ha solicitado), puede exigir alguna modalidad de interacción (el usuario debe desplazarse por el anuncio hasta alcanzar el contenido) o puede formar parte del propio contenido (aparición de determinadas marcas en vídeos o videojuegos ${ }^{15}$, véase Martín-Ibáñez, 2010).

En los tres primeros casos es habitual que se ofrezca al usuario un modo de interacción: un simple enlace con una página web adaptada al móvil, un enlace que activa una llamada telefónica, o un número corto para el envío de mensajes de texto. Este requisito de que el propio usuario deba responder supone un cambio de orientación que gana terreno frente a otras estrategias iniciales como el envío de mensajes de texto indiscriminado (Scharl; Dickinger; Murphy, 2005).

\section{"La comunicación unidireccional ya no es la única opción de la que disponen las empresas"}

\section{Volumen del mercado}

En la práctica seguida por la industria del móvil, las cifras que corresponden a las modalidades indiferenciada y orientada de la publicidad móvil se ofrecen de manera agregada muchas veces y resulta difícil separarlas. Con esta salvedad, y con las debidas precauciones que representa la medida (más aún la predicción) de un fenómeno todavía emergente, la tabla 1 resume los datos disponibles sobre la publicidad en el móvil y algunas predicciones sobre su evolución a medio plazo. De acuerdo con Feijóo-González et al. (2010) hay que señalar que el volumen de ingresos derivados de la publicidad en el móvil es aún mínimo comparado con las cifras de los sectores en los que se enmarca: consistiría en sólo alrededor del 1,3\% del volumen de negocio total generado por las comunicaciones móviles y en torno al 2,2\% del total de inversión publicitaria en 2013.

\begin{tabular}{|l|c|c|c|c|}
\hline \multicolumn{1}{|c|}{$\begin{array}{c}\text { Segmento de } \\
\text { mercado }\end{array}$} & $\mathbf{2 0 0 7}$ & $\mathbf{2 0 0 8}$ & $\mathbf{2 0 0 9}$ & $\begin{array}{c}\mathbf{2 0 1 2} \\
\mathbf{2 0 1 3}\end{array}$ \\
\hline $\begin{array}{l}\text { Publicidad basada en } \\
\text { textos e imágenes }\end{array}$ & 0,5 & 0,7 & 0,8 & $1-2$ \\
\hline $\begin{array}{l}\text { Publicidad basada en } \\
\text { web }\end{array}$ & - & 0,5 & 0,8 & $3-4$ \\
\hline $\begin{array}{l}\text { Publicidad basada en } \\
\text { vídeo }\end{array}$ & - & - & 0,5 & $1-3$ \\
\hline $\begin{array}{l}\text { Publicidad basada en } \\
\text { aplicaciones }\end{array}$ & - & - & 0,5 & $3-6$ \\
\hline Total & $0,5-1$ & $1-2$ & $2-3$ & $9-15$ \\
\hline
\end{tabular}

Tabla 1. Estimaciones de volumen de negocio mundial derivado de diversos tipos de publicidad en el móvil (millardos de €). Fuente: adaptado de Feijóo-González et al. (2010) 
En el mercado español, Madinabeitia (2010) hace referencia a los estudios que la consultora Accenture ha realizado para la MMA (Mobile Marketing Association) sobre inversión publicitaria en móviles. En el año 2008, la consultora ofrecía una cifra de 29 millones de euros, de los cuales unos 12 correspondían a inversión en medios y el resto a creatividad, tecnología, elaboración de aplicaciones y otros aspectos. En el segundo análisis, publicado en noviembre de 2009, la inversión habría aumentado de manera considerable (un 10\%), pero moderada para las expectativas creadas (un 13\%), hasta alcanzar un valor de 32 millones de euros.

Evidentemente, en la evolución del mercado de la publicidad móvil hay que considerar la respuesta de los clientes. Sin entrar en factores concretos, que actúan como impulsores o como barreras, y que serán analizados en el siguiente apartado, son interesantes los exámenes realizados sobre situaciones reales. Jayawardhena et al. (2009) deducen en su trabajo que el factor más importante de aceptación del marketing móvil es lo que denominan "confianza institucional", que englobaría la confianza en el entorno cultural, político y legal, en los medios de comunicación e incluso en la propia sociedad civil. Por su parte, Sultan y Rohm (2008), en un trabajo sobre la aceptación de la publicidad móvil en dos países (Estados Unidos y Pakistán) concluyen que el tipo de uso que se hace del móvil y el control de la publicidad (posibilidad de decidir si desean o no recibirla) son factores destacados en ambos mercados, debiendo añadirse, en el caso de Estados Unidos, la predisposición a la innovación, en general, y a las comunicaciones móviles en particular. En ambos trabajos se observan diferencias de género, siendo las mujeres menos sensibles al control de la publicidad y, pese a eso, menos dispuestas a aceptarla.

Asimismo otros análisis señalan cómo frente a otros medios, el comportamiento del usuario de la publicidad móvil puede ser diferente, respondiendo a un perfil más interactivo, social a la vez que personal, y donde la información localizada y en tiempo real juega un papel muy relevante. Así, Muoio (2009), utilizando datos de ComScore referidos a Estados Unidos, asegura que el $16 \%$ de los usuarios móviles que recibieron un anuncio por medio de un sms durante noviembre de 2008, respondieron al menos una vez, lo que supone una cifra superior a la que se obtiene en otros medios interactivos (internet y televisión digital).

Otro dato relevante lo aporta GSMA Mobile Media Metrics (Mobile Square, 2010), que proporciona datos de las páginas más visitadas en diciembre de 2009 por los casi 16 millones de usuarios de internet móvil en el Reino Unido. En total, los usuarios vieron un total de 6.700 millones de páginas y estuvieron 4.800 millones de minutos conectados durante ese mes. Pero con ser verdaderamente significativas estas cifras, la (relativa) sorpresa aparece cuando se analizan los sitios a que los usuarios dedicaron más tiempo. El dominio de las redes sociales, Facebook en particular, es abrumador con una media para cada internauta móvil del $45 \%$ del total de conexión, dato aún más destacado si se considera que el segundo puesto, que corresponde a todos los sitios web combinados de Google, llega a sólo el 8\%.

Volviendo a los Estados Unidos, el último análisis disponible del Pew Research Center (Horrigan, 2009) indica que el $26 \%$ de los adultos accede a la información sobre noticias desde un terminal móvil. Entre éstos, el $72 \%$ se interesa por el tiempo, el $68 \%$ por los titulares y la última hora, el $44 \%$ por deportes, el $35 \%$ busca información sobre el tráfico y el $32 \%$ se conecta con el propósito de tener acceso a información económica y financiera.

"El teléfono móvil puede utilizar el contexto, adaptando contenidos y aplicaciones a las condiciones particulares del usuario en ese instante"

\section{Factores clave en la evolución de la publicidad móvil}

\section{Factores que juegan a favor}

Wieland (2008) cita una serie de elementos que en su opinión condicionan el éxito de la publicidad móvil. Unos son dependientes de la evolución general de las comunicaciones móviles (número de usuarios, ancho de banda disponible, incremento del uso de medios de comunicación en el móvil) ${ }^{16}$ y otros del aprovechamiento por las empresas de las ventajas que ofrece la personalización y la ubicuidad.

Entre los del primer grupo, casi todos parecen mostrar un comportamiento favorable: las comunicaciones móviles de banda ancha son cada vez más ubicuas y asequibles, la usabilidad de los terminales ha mejorado y el precio de los terminales avanzados ha descendido, cada vez son más los segmentos de población de toda edad y condición social que se sienten cómodos utilizando el móvil y, por último, se espera una evolución rápida en el consumo de contenidos desde el móvil (Feijóo-González; Gómez Barroso, 2009).

Sin embargo, las posibilidades que ofrece el marketing personalizado son el rasgo más destacado y más prometedor como factor de éxito de la comunicación promocional en los medios móviles (Leppaniemi; Karjaluoto, 2005). Como se ha dicho, el teléfono mó- 
vil tiene la capacidad de utilizar el contexto de forma determinante, es decir, de adaptar contenidos y aplicaciones a las condiciones particulares del usuario móvil en ese instante (Pascu, 2008). Estas características podrán ser mejor aprovechadas a medida que evolucionen tecnologías cognitivas que informen del entorno físico (aprovechando sensores instalados en lugares varios, y tarjetas RFID) o del estado personal (mediante sensores que midan parámetros biológicos).

Hay otros dos factores que también deberían contribuir de manera destacada al éxito de la publicidad móvil. El primero es el progreso del comercio móvil en el que el teléfono se convierte en una "tarjeta de crédito con una antena”. En algunas compras el móvil se utiliza sólo para culminar el proceso, mientras que en otras ocasiones interviene en todas las fases. Tanto en un caso como en otro es posible introducir publicidad. Además, el seguimiento del comercio móvil proporciona, al menos potencialmente, aún más información sobre los gustos y actividades del usuario. La segunda cuestión toma el punto de vista del anunciante, para el que el móvil ofrece una ventaja adicional, que es la de permitir la medición de una forma bastante ajustada del impacto de una campaña de comunicación.

\section{Barreras y amenazas}

Dejando a un lado avances tecnológicos que puedan estar aún inmaduros, el primero de los problemas que puede impedir la evolución de la publicidad en el móvil radica en poner de acuerdo a todos los actores presentes en el ecosistema (Wilken, 2009). Efectivamente, en el mencionado proceso de aglutinación de sectores o de diferentes eslabones de la cadena de valor cada agente intenta que el valor se desplace hacia la parte que controla, aun a expensas de cuál sea la solución más eficiente. Ello puede afectar asimismo a la evolución de estándares o a la resolución de cuestiones de interfaces y de conexión entre sistemas.

\section{"La industria de la publicidad está preocupada por la posible 'saturación' de los usuarios con la publicidad móvil"}

También debe ponerse algún interrogante sobre el optimismo general que rodea el avance de las comunicaciones móviles. Frente a medios realmente universales como la televisión, en el caso del móvil generalización no significa universalización. No todo el mundo posee las habilidades (por evidentes que a los usuarios avanzados les puedan resultar) para gestionar contenidos en su móvil. La brecha digital es también, por supuesto, una cuestión de educación y de las oportuni- dades de uso de la tecnología de que se ha gozado en el pasado (Gómez-Barroso; Feijóo-González, 2006). Eso hace que ciertos colectivos puedan quedar fuera de la audiencia.

La tercera barrera se erige a partir del relativo desconocimiento de cómo adaptar los mensajes al nuevo medio (Nasco; Bruner, 2008; también Solana, 2005, que habla de la necesidad de un "nuevo lenguaje" en general para la publicidad en internet), al público a que se dirige e incluso a las circunstancias del momento. $\mathrm{Y}$ es que una misma comunicación puede ser recibida de diferente manera por dos usuarios diferentes o por el mismo usuario en dos momentos diferentes (véase el análisis de algunos de los factores que habría que considerar en Chen et al., 2009). La utilidad percibida finalmente por la audiencia es el factor determinante en su respuesta (Soroa-Koury; Yang, 2010). Esa percepción es puramente individual aunque es función de sus experiencias previas (Haghirian; Madlberger; Inoue, 2008) y también de factores culturales: Choi; Hwang y McMillan (2008) muestran diferentes grados de interacción y diferentes percepciones de valor en un experimento llevado a cabo en Corea del Sur y en Estados Unidos).

La principal amenaza se refiere a la privacidad. El teléfono móvil es considerado parte de la esfera personal de los usuarios (Aguado-Terrón; Martínez-Martínez, 2009) en mucha mayor medida que los terminales fijos. La publicidad personalizada se convierte así en un arma de doble filo. De un lado, pueden concebirse promociones realmente interesantes para quien reciba la comunicación. Pero del otro, una publicidad "demasiado" ajustada al perfil y al entorno personal puede originar justo una reacción contraria, un rechazo (Okazaki; Katsukura; Nishiyama, 2007; Peters; Amato; Hollenbeck, 2007) ${ }^{17}$. La industria de la publicidad ha expresado de hecho su preocupación por una potencialmente rápida "saturación” de los usuarios con respecto a la publicidad móvil (Muoio, 2009).

Desafortunadamente, ciertas prácticas no infrecuentes en el mercado pueden consolidar la idea de la "peligrosidad" de la publicidad móvil. En un estudio realizado en la Unión Europea en 2008 (Comisión Europea, 2008), nada menos que un $80 \%$ de los sitios web considerados que ofrecían productos como tonos y fondos de pantalla podrían no cumplir con la normativa europea de protección de los usuarios. La repercusión de los casos de fraude es, además, como sucede con frecuencia en servicios novedosos, particularmente notoria $^{18}$.

Si como consecuencia de estos incidentes la legislación se endurece, la necesidad de cumplir con unas normas demasiado exigentes podría ser también un 
obstáculo para la evolución de la publicidad móvil. El ordenamiento aplicable puede ser diferente dependiendo del servicio o aplicación concreta. En algunos casos la legislación puede exigir un sistema de aceptación previa para recibir publicidad.

\section{"El teléfono móvil será la herramienta con la que se satisfaga la mayor parte de las necesidades de comunicación"}

\section{Conclusiones}

La comunicación unidireccional ya no es la única opción de que disponen las empresas, y no es desde luego la más interesante. Los avances tecnológicos permiten crear situaciones en las que el mensaje que se envía al receptor es suficientemente personal como para que, idealmente, exista una respuesta de éste que convierta en bidireccional la comunicación.

Algunas estrategias para lograrlo han sido ya exploradas en el internet tradicional, pero existe otro, el internet móvil que va ganando terreno a toda velocidad. Y este es sólo el principio de un proceso que llevará al teléfono móvil a ser la herramienta con que se satisfaga la mayor parte de las necesidades de comunicación del individuo. El móvil posee unas características únicas (personal, se lleva siempre encima, ofrece información de contexto) que permitirán ir, en el terreno de la comunicación y de la publicidad, mucho más allá de lo que se ha experimentado.

Con todo, la publicidad móvil se halla en un estado incipiente de evolución y ha recibido poca atención de las empresas, que no acaban de apostar por un medio que no conocen bien. Pero se trata posiblemente de una cuestión de tiempo. Los factores para el crecimiento de la publicidad móvil parecen sólidos aunque no hay que perder de vista todos los elementos que se han descrito y que pueden obstaculizar o retrasar su implantación.

Como en todo terreno por explorar, el pionero tiene más difícil el avance pero mucha mayor recompensa que obtener. Aquellas empresas que primero sean conscientes de la potencialidad del móvil como vehículo para su comunicación y lleven a cabo estrategias innovadoras e ingeniosas podrían obtener una ventaja indudable sobre sus competidores. El premio no parece pequeño para cualquier empresa en unos tiempos como los actuales en que la batalla en costes parece difícil de ganar y es el uso inteligente de la promoción y la comunicación una de las variables básicas para permanecer en el mercado.

\section{Agradecimientos}

Este artículo forma parte de los trabajos del proyecto de investigación Evolución del medio móvil en Espa$\tilde{n} a$ (CSO2009-07108). Asimismo se basa parcialmente en el trabajo de uno de los autores para el Institute for Prospective Technological Studies de la Comisión Europea sobre el futuro impacto de las aplicaciones y contenidos móviles.

\section{Notas}

1. Publicidad no debe identificarse con marketing. Para Balmer y Greyser (2006) la comunicación empresarial formaría, junto con la imagen, reputación, identidad y marca, el marketing corporativo.

2. Se calcula que en España cada persona recibe diariamente, en promedio, más de mil impactos publicitarios (Solana, 2005). Peor aún, la publicidad está mal vista socialmente y no se le reconoce la importancia de su aportación a la economía (Madinabeitia, 2010).

3. Todo tipo de dispositivos transportables: teléfonos pero también pequeños ordenadores o agendas electrónicas con diferentes características. En adelante se utilizará con frecuencia "el móvil" como expresión que sustituye a esta definición más exacta.

4. Los últimos datos disponibles señalan claramente esta tendencia. Entre los más recientes, el último informe Mediascope Europe, realizado en 2010 por la Asociación Europea de Publicidad Interactiva (EEIA) en 4.000 hogares del Reino Unido, Alemania, Francia y España, señala que en España se dedica ya más tiempo a internet móvil (5,5 horas semanales) que a leer el periódico (4,6 horas) o revistas (3,6 horas). Asimismo, de los usuarios españoles con un teléfono con conexión a internet el $74 \%$ se conecta a redes sociales, el $82 \%$ realiza algún tipo de búsqueda y el $85 \%$ utiliza el correo electrónico al menos una vez al mes.

5. También para abreviar, "publicidad móvil” sustituirá a "publicidad difundida mediante los contenidos, servicios y aplicaciones que se reciben o utilizan en terminales móviles".

6. Poco exitosas. La iniciativa más destacable fue la del operador británico $B l y k$, que ofrecía minutos de voz gratis a cambio de interrumpir periódicamente la conversación para introducir publicidad, además de enviar diversos tipos de publicidad al terminal móvil. Su objetivo declarado era el segmento de población entre 16 y 24 años. En la actualidad está redefiniendo su modelo de negocio.

7. Apple, con su plataforma iTunes-iPhone-App Store, o Nokia que ha seguido los mismos pasos con Symbian y Ovi. Pero también Google y su sistema operativo abierto para móviles Android, Blackberry de RIM, Microsoft con Windows Mobile y una larga lista a la que se han sumado recientemente otras empresas como Samsung y Sony.

8. Quizá el ejemplo más destacado de esta estrategia de control de la plataforma sea Apple, con su terminal iPhone y su tienda de aplicaciones App Store. Y efectivamente, siguiendo está lógica ha anunciado (febrero de 2010) un módulo específico para la inclusión de publicidad en aplicaciones dentro de su herramienta de elaboración de software para su plataforma.

9. Google ha apostado particularmente por la relación entre publicidad y posición del usuario, augurando que los "servicios locales" serán una significativa fuente de ingresos. Google es además la compañía propietaria de la patente EUA número 7668832, recientemente concedida a partir de una solicitud realizada originalmente en abril de 2004, que le otorga los correspondientes derechos sobre el uso de la información de posición para "entregar" al usuario un determinado anuncio.

10. Prueba del interés en manejar esta información es que en diversos mercados los operadores de comunicaciones móviles han aceptado que una empresa independiente contabilice y presente (de manera anónima) los datos básicos de uso de internet móvil, de forma que puedan ser aprovechados por anunciantes y proveedores. Este es por ejemplo el caso del Reino Unido donde ComScore ha comenzado a realizar esta tarea desde finales de 2009 poniendo de acuerdo ni más ni menos que a Vodafone, O2 (Telefónica), Orange (France Telecom), T-Mobile (Deutsche Telecom) y $3 U K$.

11. Una práctica típica en este segmento es el envío de los llamados “cupones móviles” que ofrecen al usuario un código que le proporciona por ejem- 
plo algún descuento en una cierta compra. Entre las muchas compañías que trabajan en este campo se puede consultar por ejemplo iLoop Mobile.

12. Jansen, Brown y Resnick (2007) confirman que los enlaces patrocinados son percibidos por los usuarios como publicidad y, en ese sentido, menos preferidos que el resto de resultados obtenidos en la búsqueda, a no ser que los perciban como realmente relevantes. Por supuesto, el líder de este segmento es Google que estima que en breve el negocio derivado de las búsquedas móviles será mayor que el de internet convencional. En las búsquedas en móvil, los contenidos presentados al usuario son habitualmente adaptados a la pantalla limitada (reducción del número de resultados de la búsqueda, limitación del número de palabras clave de los enlaces patrocinados, etc.).

13. Entre los contenidos, quizá el vídeo sea el campo con más posibilidades de consumo a cambio de publicidad, siguiendo un esquema conceptualmente similar al de la televisión convencional. YouTube en su versión móvil es un ejemplo elocuente de compañía que pretende beneficiarse de este esquema. Por su parte, Mywaves ofrece estos servicios de publicidad a las marcas $\mathrm{y}$ anunciantes interesados.

14. Las aplicaciones (los juegos son el ejemplo principal) se pueden descargar gratuitamente a cambio de publicidad antes y durante el uso de la aplicación. Entre las muchas empresas dedicadas a este segmento se puede citar como ejemplo relevante Greystripe.

15. Este es el llamado advergaming del que existen numerosos ejemplos, como el de la compañía Unkasoft.

16. Según el mencionado informe Mediascope Europe de la EIAA, $121 \mathrm{mi}$ llones de europeos usaban banda ancha inalámbrica a finales de 2009. De ellos, 10 millones lo hacen en España, con un incremento del $11 \%$ con respecto al año anterior.

17. Sultan y Rohm (2008) encuentran en su trabajo mayores niveles de preocupación por las cuestiones relacionadas con la privacidad en un mercado maduro (Estados Unidos) que en un mercado emergente (Pakistán).

18. En 2005 hubo un escándalo con la empresa Jamba, que parece ser que cargaba cantidades adicionales a los usuarios, en ocasiones sin que éstos fueran conscientes, al ofrecer otros tonos tras la adquisición de un primero. El Mail on Sunday en el Reino Unido habló de la "estafa de los tonos" (ringtone rip-off).

http://www.mailonsunday.co.uk/pages/live/articles/news/news.html?in article_id $=345213 \&$ in_page_id $=1770$

\section{Bibliografía}

Aguado-Terrón, Juan-Miguel; Martínez-Martínez, Inmaculada J. “De la web social al móvil 2.0: el paradigma 2.0 en el proceso de convergencia mediática de la comunicación móvil". El profesional de la información, 2009, v. 18 , n. 2, pp. 155-161.

Argenti, Paul A. "Corporate communication as a discipline: toward a definition”. Management communication quarterly, 1996, v. 10, n. 1, pp. 73 97.

Balmer, John M. T.; Greyser, Stephen A. "Corporate marketing: integrating corporate identity, corporate branding, corporate communications, corporate image and corporate reputation". European journal of marketing, 2006, v. 40, n. 7-8, pp. 730-741.

Chen, Peng-Ting; Hsieh, Hsin-Pei; Cheng, J. Z.; Lin, Yu-Sheng. "Broadband mobile advertisement: what are the right ingredient and attributes for mobile subscribers". En: Proc of the Portland Intl Conf on Management of Engineering \& Technology. Portland: IEEE Publishing, 2009, pp. 625-632

Choi, Yu-Kyun; Hwang, Jang-Sun; McMillan, Sally J. "Gearing up for mobile advertising: a cross-cultural examination of key factors that drive mobile messages home to consumers". Psychology and marketing, 2008, v. 25 , n. 8 , pp. $756-768$.

Comisión Europea. EU crackdown of ringtone scams. Frequently asked questions. 2008.

http://europa.eu/rapid/pressReleasesAction.do? reference $=M E M O / 08 /$ $516 \&$ format $=H T M L \&$ aged $=0 \&$ language $=E N \&$ guiLanguage $=e n$

Del-Pino-Romero, Cristina. "Internet y los nuevos consumidores. El nuevo modelo publicitario". Telos, 2010, n. 82.

Feijóo-González, Claudio; Gómez-Barroso, José-Luis. "Factores clave en los mercados de acceso móvil a contenidos". El profesional de la información, 2009, v. 18, n. 2, pp. 145-154.

Feijóo-González, Claudio; Maghiros, Ioannis; Bacigalupo, Margherita; Abadie, Fabienne; Compañó, Ramon; Pascu, Corina. Content and applications in the mobile platform: on the verge of an explosion. Sevilla: Institute for Prospective Technological Studies, 2010. En prensa.

Fransman, Martin. The new ICT ecosystem. Implications for Europe. Edinburgh: Kokoro, 2007.

Gómez-Barroso, José-Luis; Feijóo-González, Claudio. "Public policies against the digital divide: a necessary adaptation to different degrees of development". Intl journal of internet and enterprise management, 2006, v. 4, n. 3 , pp. $257-268$

Haghirian, Parissa; Madlberger, Maria; Inoue, Akihiro. "Mobile advertising in different stages of development: a cross-country comparison of consumer attitudes". Proc of the 41st Annual Hawaii Intl Conf on System Sciences. Hawaii: IEEE Publishing, 2008, pp. 48-49.

Horrigan, John. Wireless internet users. Washington DC: Pew Research Center's Internet \& American Life Project. 2009.

http://pewinternet.org/Reports/2009/12-Wireless-Internet-Use.aspx

Jansen, Bernard J.; Brown, Anna; Resnick, Marc. "Factors relating to the decision to click on a sponsored link". Decision support systems, 2007, v. 44 , n. 1 , pp. $46-59$.

Jayawardhena, Chanaka; Kuckertz, Andreas; Karjaluoto, Heikki; Kautonen, Teemu. "Antecedents to permission based mobile marketing: an initial examination". European journal of marketing, 2009, v. 43, n. 3-4, pp. 473-499.

Leppaniemi, Matti; Karjaluoto, Heikki. "Factors influencing consumers' willingness to accept mobile advertising: a conceptual model". Intl journal of mobile communications, 2005, v. 3, n. 3, pp. 197-213.

Madinabeitia, Eduardo. "La publicidad en medios interactivos. En busca de nuevas estrategias". Telos, 2010, n. 82.

Martín-Ibáñez, Eva. "Videojuegos y publicidad. Cómo alcanzar a las audiencias que escapan de los medios tradicionales". Telos, 2010, n. 82

Melewar, Tengku C.; Karaosmanoglu, Elif. "Seven dimensions of corporate identity: a categorisation from the practitioners' perspectives". European journal of marketing, 2006, v. 40, n. 7-8, pp. 846-869.

MMA. Mobile applications. Mobile Marketing Association, 2008. http://www.mmaglobal.com/mobileapplications.pdf

Mobile squared: Thinking about mobile, 2010, n. 7. http://www.d2mobile.co.uk/uploads/news/issue_7_feb.pdf

Muoio, Kevin. Key trends in mobile content usage \& mobile advertising. ComScore, 2009

http://microsoftpanel.com/Press_Events/Presentations_Whitepapers/2009/ Key_Trends_in_Mobile_Content_Usage_Mobile_Advertising

Nasco, Suzanne A.; Bruner, Gordon C. "Comparing consumer responses to advertising and non-advertising mobile communications". Psychology and marketing, 2008, v. 25, n. 8, pp. 821-837.

Okazaki, Shintaro; Katsukura, Akihiro; Nishiyama, Mamoru. "How mobile advertising works: The role of trust in improving attitudes and reca11". Journal of advertising research, 2007, v. 47, n. 2, pp. 165-178.

Pascu, Corina. An empirical analysis of the creation, use and adoption of social computing applications. Sevilla: Institute for Prospective Technological Studies, 2008. Document EUR 23415 EN.

http://ipts.jrc.ec.europa.eu/publications/pub.cfm?id=1684

Peters, Cara; Amato, Christie H.; Hollenbeck, Candice R. "An exploratory investigation of consumers' perceptions of wireless advertising". Journal of advertising, 2007, v. 36, n. 4, pp. 129-145.

Scharl, Arno; Dickinger, Astrid; Murphy, Jamie. "Diffusion and success factors of mobile marketing". Electronic commerce research and applications, 2005, v. 4, n. 2, pp. 159-173.

Solana, Daniel. “Anuncios en internet. La nueva publicidad”. Telos, 2005 n. 64 , pp. $74-78$.

Soroa-Koury, Sandra; Yang, Kenneth C. C. "Factors affecting consumers" 
responses to mobile advertising from a social norm theoretical perspective". Telematics and informatics, 2010, v. 27, n. 1, pp. 103-113.

Sultan, Fareena; Rohm, Andrew J. "How to market to generation M(obile)". MIT Sloan management review, 2008, v. 49, n. 4, pp. 34-41.

Wieland, Ken. "Mobile advertising reality check". Telecommunications (Americas edition), 2008, v. 42, n. 1, p. 8.

Wilken, Rowan. "'Waiting for the kiss of life': mobile media and advertising". Convergence: The international journal of research into new media technologies, 2009, v. 15, n. 4, pp. 427-445.

Claudio Feijóo-González. CeDInt, Universidad Politécnica de Madrid. Campus de Montegancedo, 28223
Pozuelo de Alarcón, Madrid.

cfeijoo@cedint.upm.es

José-Luis Gómez-Barroso. Dpto. Economía Aplicada e Historia Económica, UNED. Po Senda del Rey, 11, 28040 Madrid.

jlgomez@cee.uned.es

Inmaculada J. Martínez-Martínez. Dpto. de Información y Comunicación, Facultad de Comunicación y Documentación, Universidad de Murcia. Campus Universitario de Espinardo. 30100 Murcia.

inmartin@um.es

\section{Máster Oficial Universitario CALSI}

\section{Objetivos}

Especializar a profesionales de la información en la gestión de contenidos a través de diferentes plataformas para todos los ámbitos de la sociedad.

Ahondar y ampliar los conocimientos en Archivística y Documentación con un enfoque dirigido a la aplicación de las tecnologías de la información en sus nuevos canales.

\section{Especialidades}

E-consulting en la sociedad de la información

Procesos informativos en las organizaciones. Normativa relativa a los contenidos y su distribución. Sistemas de información en las empresas. Normas y recomendaciones sobre tratamiento y difusión de datos.

\section{Administración electrónica}

Implantación de la administración electrónica desde las oficinas administrativas. Sistematización de los trámites electrónicos y puesta en marcha de un sistema de gestión documental para la administración electrónica a partir de la legislación vigente.

\section{Servicios y contenidos web}

Técnicas aplicadas a la gestión de contenidos en diversos formatos y distribuidos en multiplataforma. Desarrollo de servicios de información en línea.

\section{Estructura del Máster}

75 ECTS a impartir en dos cursos académicos. Horario de tarde

Materias:

42,5 ECTS - se compone de asignaturas comunes y asignaturas de la especialidad escogida Asignaturas de libre configuración curricular: 17,5 ECTS

Tesina fin de Máster:

15 ECTS

\section{Profesorado}

Este Máster será impartido por profesorado de diversas universidades nacionales así como profesionales de reconocido prestigio.

\section{Plazos orientativos}

Periodo de preinscripción Primera quincena de mayo Periodos de matrícula:

Segunda quincena de julio Primera quincena de septiembre

\section{Información}

Secretaría del Departamento de Comunicación Audiovisual, Documentación e Historia del Arte (DCADHA) de la UPV.

Teléfono: 963877390

e-mail: dephar@upvnet.upv.es Página web:

http://www.upv.es/miw/infoweb/po/mas/27/ind ex2005c.html

\section{Preinscripción}

http://www.upv.es/contenidos/PO/menu_4950 $35 \mathrm{c} . \mathrm{html}$

Estos estudios dan acceso al programa de Doctorado. El máster admite estudiantes con titulación universitaria oficial española o de la Unión Europea, así como titulados universitarios de países no pertenecientes a la UE previa comprobación de la equivalencia del nivel de formación a un título universitario español.

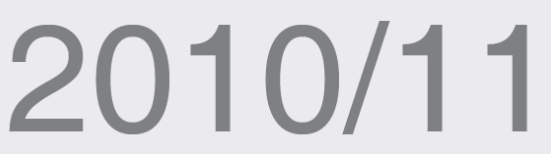

UNIVERSIDAD

POLITECNICA

DE VALENCIA
MINISTERIO

DE EDUCACIÓN

Y CIENCIA 